\title{
Facts of research on protein-protein interactions
}

\author{
Youhe Gao \\ Gene Engineering and Biotechnology Beijing Key Laboratory, Department of Biochemistry and Molecular Biology, Beijing Normal University, \\ Beijing 100875, China
}

Received September 29, 2016; accepted October 11, 2016; published online November 4, 2016

Citation: Gao, Y. (2017). Facts of research on protein-protein interactions. Sci China Life Sci 60, 313-314. doi: 10.1007/s11427-016-0292-0

Dear Editor,

Fact one of the current research on protein-protein interactions is that tagging is widely used. Tagging is a good way to track or visualize a protein. Researchers assume that tagging does not change the structure and function of the protein too much. This is probably not true for many proteins.

Fact two is that overexpression is widely used too. To introduce an exogenous tagged protein into the cell, overexpression is the most common method. Investigators assume that overexpression does not interfere with the location and interactions of the protein in question. This is possibly not true for many proteins either.

Fact three is that researchers believe that they wash the protein complex gently enough (so that the weak interactions remain intact) but hard enough so that no new false positive interaction can form and stay. This is probably not true in most cases.

Fact four is that many protein interactions have been identified under conditions other than their natural environment. The proteins were forced to be expressed and to interact in a test tube in an artificial solution, in other cell types, in the nucleus, on the membrane, or on the pili of a phage.

Fact five is that many large-scale studies that are widely cited have been conducted by the methods with one or more of the above flaws. When an interaction is found in two independent studies, it is believed to be a scientific fact.

There is actually a way to get the facts on protein inter-

gaoyouhe@bnu.edu.cn action (Figure 1). Fast Fixation is necessary to study real-time protein-protein interactions under physiological conditions. Fast formaldehyde crosslinking can fix transient and weak protein interactions. With brief exposure to a high concentration of formaldehyde during the crosslinking, the complex is crosslinked only partially, so that the complex is small enough to be resolved by SDS-PAGE, and the uncrosslinked parts of the proteins can be used for identification by shotgun proteomics. Immunoaffinity purification can Fish out complexes that include the proteins of interest. Because the complex is covalently bound, it can be washed as harshly as the antibody-antigen reaction can stand; the weak interactions will remain. Even if the nonspecific binding can persist on the beads or antibody, it will be eliminated at the next step. To Filter out these complexes, SDS-PAGE is used to disrupt non-covalent bonds, thereby eliminating uncrosslinked complexes and simultaneously providing molecular weight information for identification of

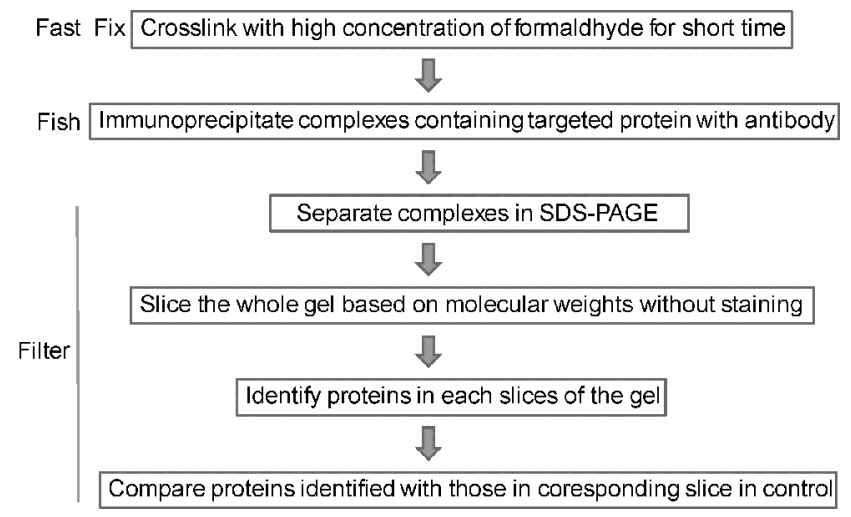

Figure 1 4F-acts Strategy 
the complex. The SDS-polyacrylamide gel can then be sliced on the basis of the molecular weight without staining. All the protein complexes can be identified with the sensitivity of mass spectrometry rather than sensitivity of the staining method. This strategy was originally named $4 \mathrm{~F}$-acts before a reviewer changed the name to $4 \mathrm{~F}$, for fail (1).

The advantages are the following: (i) The method does not involve tagging. (ii) It does not include overexpression. (iii) A weak interaction can be detected because the complexes can be washed as hard as the antigen-antibody reaction can stand as the complexes are crosslinked covalently. No new covalent bond can form as a false positive result. (iv) The formaldehyde crosslinking can be performed at the cellular, tissue, or organ level fast enough so that the protein complexes are fixed in situ in real time.
A large-scale study should be conducted to evaluate this strategy and give investigators the facts of real protein interactions in situ in real time.

Compliance and ethics The author(s) declare that they have no conflict of interest.

Acknowledgements This work was supported by the National Key Research and Development Program of China (2016YFC1306300), the National Basic Research Program of China (2013CB530850), and funds from Beijing Normal University (11100704, 10300-310421102).

Zhu, L., Li, M., Wei, L., Liu, X., Yin, J., and Gao, Y. (2014). Fast fixing and comprehensive identification to help improve real-time ligands discovery based on formaldehyde crosslinking, immunoprecipitation and SDS-PAGE separation. Proteome Sci 12, 6.

Open Access This article is distributed under the terms of the Creative Commons Attribution License which permits any use, distribution, and reproduction in any medium, provided the original author(s) and source are credited. 